\title{
Retrasos del desarrollo psicomotriz en niños y niñas urbanos de 0 a 5 años: Estudio de caso en la zona urbana de Cuenca, Ecuador
}

\author{
Lourdes Huiracocha T., Gladys Robalino I., Miriam S. Huiracocha T., Jorge L. García A., Carmen \\ G. Pazán T., Aydeé Angulo
}

Facultad de Ciencias Médicas, Universidad de Cuenca, Cuenca, Ecuador

Autor para correspondencia: lourdes.huiracocha@ucuenca.edu.ec

Fecha de recepción: 23 de febrero 2012 - Fecha de aceptación: 21 de mayo 2012

\section{RESUMEN}

Este estudio transversal se realizó en el período de enero a junio de 2009 en niños y niñas de 0 a 5 años de edad para determinar la prevalencia de los retrasos del desarrollo psicomotor. La población infantil del estudio perteneció a los Centros de Desarrollo Infantil fiscales, privados, regulares, especiales urbanos de Cuenca. Se clasificó a los niños con retrasos en -con discapacidad y sin discapacidad-, registrando cuántos se beneficiaron de la atención temprana. Se buscó la asociación del retraso con desnutrición, microcefalia, familia no nuclear, migración de los padres y cuidadora no materna. La muestra probabilística aleatoria por estratos se calculó en EpiInfo ${ }^{\mathrm{TM}}$, con $5 \%$ de frecuencia de retraso, $3 \%$ de error de inferencia, $95 \%$ de confianza y $10 \%$ de pérdidas $(\mathrm{N}=8537, \mathrm{n}=$ 462). Siete equipos de especialistas con el test de Brunet-Lezine diagnosticaron primero el retraso y luego la discapacidad usando la Escala de Valoración de la Situación de Dependencia de 0 a 3 años y con Baremo de la Situación de Dependencia de 3 a 5 años. La desnutrición se identificó midiendo y ubicando la antropometría en desvíos estándar en las tablas de la Organización Mundial de la Salud. Las otras variables se indagaron por encuestas. El estudio reveló que el 11\% (IC95\%, 8 al 14\%) presentó retraso, $6 \%$ sin discapacidad y $5 \%$ con discapacidad, el $31 \%$ se benefició de la atención temprana. Se encontró relación de retraso del desarrollo psicomotor con desnutrición $(p<0,001)$, microcefalia $(\mathrm{p}<0,002)$ y con familia no nuclear $(\mathrm{p}<0,005)$. No así con cuidadora no materna, ni con migración. Estos datos orientan a implementar tempranamente programas de intervención.

Palabras clave: Desarrollo psicomotriz, discapacidad, niños y niñas, estado nutricional, microcefalia, migración, familia.

\begin{abstract}
A cross-sectional survey was conducted in the period January-June 2009 on children in the age group 0 to 5 years to determine the prevalence of delays in psychomoter development. The surveyed infancy population belonged to public, private, regular and special Child Development Centers of the City of Cuenca. Delays in psychomoter development with and without disabilities were recorded, together with the number of children that benefited from early care taking. Observations were related to the level of malnutrition, failure of brain growth, the family composition, the migration of parents and the replacement caregiver by mother-absence. The stratified random probability sample was calculated with EpiInfo ${ }^{\mathrm{TM}}$ with 5\% frequency delay, 3\% error of inference, $95 \%$ confidence and $10 \%$ loss $(\mathrm{N}=$ $8537, \mathrm{n}=462$ ). Seven teams of specialists diagnosed first the delay in psychomoter development using the Brunet-Lezine test, followed by measuring the disability of the 0 to 3 year old children using the Situation Dependency Valorization Scale, and the Situation Dependency Rating Scale for the 3 to 5 year olds. The malnutrition status was assessed measuring the anthropometry expressed in number of standard deviations with reference to the WHO growth charts. All other variables were recorded on the basis of surveys. The study revealed that $11 \%$ (IC95\%, 8 to 14\%) demonstrated a delay in psychomotor development, $6 \%$ without disability and 5\% with disability, and $31 \%$ benefited from early intervention. A relationship between delayed psychomotor development with malnutrition ( $\mathrm{p}<$
\end{abstract}


$0,001)$, microcephaly $(\mathrm{p}<0,002)$ and non-nuclear family $(\mathrm{p}<0,005)$ was found. Neither the replacement caregiver by mother-absence, nor the migration of the parents, seems to be causal factors. Results justify the establishment of early intervention programs.

Keywords: Psychomotor development, disability, boys and girls, nutritional status, microcephaly, migration, family.

\section{INTRODUCCIÓN}

Las leyes y políticas ecuatorianas establecen que todos los niños, niñas y adolescentes (NNA) tienen derecho al desarrollo integral e implementan programas y proyectos en los ámbitos de educación, salud, vivienda, seguridad social, cultura, comunicación para garantizar este derecho. También se instituyen políticas específicas para responder con atención de calidad a las necesidades especiales de los NNA con problemas del desarrollo psicomotor con o sin discapacidad con el fin de lograr una vida digna para ellos y sus familias (Secretaría Técnica del Frente Social, 2004; Concejo de la Niñez y Adolescencia, 2007; Asamblea Constituyente, 2008). Sin embargo, la protección de los derechos de los niños y niñas con retrasos del desarrollo con o sin discapacidad no se cumplen por: (i) no son detectados, ni diagnosticados, ni intervenidos, ni incluidos; (ii) no hay un registro estadístico real del número de estos NNA lo que minimiza el problema haciendo que no se instituyan programas ni recursos financieros necesarios; y (iii) no se ha documentado con investigaciones los factores de riesgo relacionados con retraso y con discapacidad lo que imposibilita la prevención. Esto hace que estos niños y niñas deterioren su condición neurobiológica-social y vayan a la discapacidad o a grados más severos de ella. La sociedad los convierte en seres excluidos, confinados en un espacio y alejados de la convivencia. Según el Instituto Nacional de la Niñez y de la Familia (INNFA ${ }^{1}$ ), el Estado no ha viabilizado programas efectivos que aborden esta problemática (Polit, 2008).

\subsection{El desarrollo psicomotor, retraso, discapacidad}

El desarrollo es la adquisición dinámica y compleja de capacidades funcionales en los sistemas: evolutivo, sensorial, afectivo-conductual, sexual, social, que posibilitan una equilibrada interacción con el mundo circundante. La evolución del desarrollo requiere de la maduración y plasticidad del sistema nervioso, de una estructura genética y la satisfacción integral de las necesidades (educación, vivienda, alimentación, diversión, familia, amor, solidaridad, generosidad) capacidades, aspiraciones (Peñafiel y col., 2003; Culbertson y col., 2003).

Sin embargo, las condiciones socioeconómicas de pobreza del país establecen circunstancias que hacen que el desarrollo no pueda seguir su curso normal o no pueda ser cuidado adecuadamente. Los retrasos del desarrollo con o sin discapacidades son fruto de la sumatoria de factores de riesgo social, ambiental y biológicos (Ministerio de Trabajo de Asuntos Sociales de España, 2003; Observatorio Social del Ecuador, 2006; UNICEF, 2008). El diagnóstico y la intervención por la plasticidad del sistema nervioso central, hace que la evolución y el pronóstico sean más favorables o que causen menos complicaciones. El retraso del desarrollo es una demora en la adquisición de las funciones de alguna o de todas las áreas del desarrollo, de acuerdo a la edad y sexo que pueden o no causar discapacidad (Narbona y Schlumberger, 2008). La discapacidad es la limitación en la actividad y restricción en la participación, originada por un trastorno que afecta en forma permanente (Centro de Atención a la Infancia y Familia, 1996; OMS, 2001; Consejo Nacional de discapacidades y col., 2005). La bibliografía internacional determina una variada frecuencia de retrasos del desarrollo entre el 16 y $18 \%$ en niños y niñas (Avaria, 2005).

Un estudio en Madrid (España), realizado en edades comprendidas entre 0 y 18 años determinó el 12,7\% de prevalencia (Aláez y col., 1998). En Argentina con la Prueba Nacional de Pesquisa de

\footnotetext{
${ }^{1}$ INNFA es el acrónimo del Instituto Nacional de la Niñez y de la Familia antes del 2009. Desde del año 2009 la abreviación de este instituto se cambió por INFA.
} 
trastornos inaparentes del desarrollo psicomotor en el primer nivel de atención se encontró en 839 niños y niñas presuntamente sanos que concurrían a tres centros de salud, el $20 \%$ de retrasos (Oiberman y col., 2006; PRUNAPE, 2008). En el 2005 la UNICEF aplicó un módulo de discapacidad en la Encuesta de Indicadores Múltiples por Conglomerados (MICS) en 191.199 niños de 2 a 9 años de edad de 18 países, reveló que el promedio conglomerado de niños y niñas con discapacidad es del $23 \%$ pero existen grandes variaciones entre los países, así es el $2 \%$ en Uzbekistán al 35\% en Djibouti (UNICEF, 2010). Diferentes censos realizados en los países han tratado de identificar a las personas con discapacidad, así la frecuencia en el 2004 en Chile fue del 12,93\% (Gobierno de Chile, 2004), en Argentina del 7,1\% y en Uruguay del 7,6\% (Organización Panamericana de la Salud, 2010).

En el Ecuador no hay estudios de prevalencia de los retrasos del desarrollo. La Facultad Latinoamericana de Ciencias Sociales de FLACSO indica que en el país hay 989 instituciones que trabajan con NNA con capacidades especiales pero no se sabe cuántos. Fuentes generadoras de información como el INEC (Instituto Nacional de Estadística y Censo), Sistema de Información para la Infancia (SIPI) tampoco disponen de registros suficientes de datos de niños, niñas con retrasos del desarrollo (Larrea, 2008). En el Centro de Desarrollo Infantil de la Universidad de Cuenca (CEDIUC), que atiende a niños y niñas de 0 a 6 años de edad con sospecha de alteración del crecimiento y desarrollo, en el año 2002, se determinó que de 284 niños y niñas, el $75 \%$ tuvieron un desarrollo normal y el $25 \%$ presentaron retraso del desarrollo psicomotor, las causas más frecuentes fueron Síndrome de Down, Parálisis Cerebral Infantil, déficit intelectual e hipoacusia (Huiracocha y col., 2004). Según el CONADIS (2005) el 12,14\% del total de la población ecuatoriana son personas con discapacidad y de estos el $1,7 \%$ son niños o niñas; sin embargo, se piensa que son más pero que no están identificados ni registrados ya sea por falta de programas de atención que los detecte o porque sus padres al no aceptarlos no los presentan como tal.

\subsection{Factores asociados a retrasos del desarrollo}

Varios estudios han demostrado como la desnutrición impacta sobre el desarrollo directamente proporcional al grado y a la duración de la misma (Lahiri y col., 1994); desde lesiones drásticas con examen neurológico anormal a problemas escolares con examen neurológico normal (Perales y col., 1996). Los cambios en el sistema nervioso central son alteraciones en la mielinización de las fibras, modificaciones neuronales y de los tejidos a nivel histológico, electrofisiológico, bioquímico y conductual. Déficits en la cantidad de ADN, fosfolípidos, esfingomielina, proteínas nucleares y en algunos neurotransmisores. El músculo y el desarrollo locomotor también presentan alteraciones secundarias a la desnutrición. Problemas visuales, déficits atencionales y de la memoria (Upadhyay y col., 1989; Bhoomika y col., 2008). Investigaciones en América Latina, África y Estados Unidos han demostrado que niños desnutridos tienen un menor Coeficiente Intelectual (CI) que niños normales del mismo nivel socioeconómico (Mansur y Neto, 2006), el estudio realizado en Sarlahi, Nepal, del 2007 al 2008 en niños de 1 a 9 años de edad determina (OR de 1.47; IC95\%: 1,02 a 2,12) que la desnutrición es un factor de riesgo para el retraso del desarrollo (Wu y col., 2010). En la India, un estudio utilizando tareas de desarrollo cognitivo reveló un rendimiento más bajo en niños desnutridos que en controles, aún controlando variables socioeconómicas (Reyes y col., 1990). A nivel mundial, el estudio de la UNICEF con MICS del 2005-2006, determina que existe relación entre el retraso del desarrollo y retardo del crecimiento pero solicita mayores estudios en esta área (Gottlieb y col., 2009).

El Informe del 2009 del Subcomité de estándares de calidad de la Academia Americana de Neurología, el Comité de Prácticas de la Sociedad de Neurología Infantil y los protocolos neurológicos indican que los niños con microcefalia presentan mayor riesgo de sufrir alteraciones del desarrollo (Fejerman y Fernández, 1998; Ashwal y col., 2009; Martí y Cabrera, 2010).

Los factores sociales de familia disfuncional, presencia de migración de los padres, tipo de cuidador (madre u otro miembro) están relacionados con el desarrollo psicomotor de los niños. La familia porque de acuerdo a como se ejerzan las funciones (asegurar la supervivencia, garantizar la protección, el afecto y la identidad de sus seres) es como se puede o no potenciar el desarrollo del niño o niña (Bringiotti, 2005; Quintero, 2007). En los últimos decenios las estructuras familiares se han modificado por diferentes causas (participación de la mujer en el campo laboral, migración, divorcio, nuevas concepciones de convivencia), hay más hogares con la presencia de un solo padre, 
familias en la que el cuidado del niño o niña está a cargo de los abuelos, tíos u otros (Nelson y Varga, 2001). Según la Comisión Económica para América Latina y el Caribe (CEPAL) entre el período de 1990 y 2005 las familias nucleares se han reducido del 46,3\% a 41,1\% (CEPAL, 2007). Según la primera Encuesta Nacional de la Niñez y Adolescencia (ENNA) en Ecuador, del 2010 la mitad de las familias son nucleares, el $10 \%$ son monoparentales, el 35\% extendidos, el 5\% son hogares compuestos (Observatorio de los Derechos de la Niñez y Adolescencia, 2010). En Cuba se realizó un estudio descriptivo prospectivo del desarrollo psicomotor y funcionamiento familiar y se encontró que el $75 \%$ de los niños con retraso del desarrollo psicomotor vivían en familias disfuncionales (Robaina y Rodríguez, 2000). Un estudio descriptivo-correlacional brasileño de 2007 en 120 niños y niñas de 6 a 44 meses determinó la frecuencia del 33\% de "cuidado en el desarrollo" en vez de retraso y de estos el $88 \%$ estuvo cuidado por la madre (18\% solteras), el $12 \%$ por los abuelos con edades entre 68 y 77 años de edad (Santa María y Linhares, 2007). Un estudio de la UNICEF y el Gobierno del Distrito Federal realizado en México del 2001 al 2003 en 6.626 NNA con discapacidad hasta los 18 años, encontró que entre el 55 y $62 \%$ tienen familias nucleares, en las familias que no son nucleares predominó básicamente las familias expandidas donde al padre y a la madre se suman los abuelos o los tíos. Entre quienes viven una situación de marginación muy alta no aparecen familias expandidas porque se trata de migrantes que llegan a la ciudad separándose de sus familias de origen. En este nivel es más alto el porcentaje de familias monoparentales, integradas básicamente por madres solteras $(19,4 \%)$, aparece además, una forma de familia que surge de la solidaridad social de los más vulnerables, la familia compuesta en la que se integran familiares, amigos y vecinos (Gobierno del Distrito Federal y UNICEF, 2006).

La migración ocasiona que los niños se queden bajo el cuidado de terceras personas que pueden ser familiares o no; cuidador que tiene influencia en la formación de la personalidad, identidad, autoestima del niño o niña (ONU, 2002). Los padres emigran al exterior dejando a sus niños, en muchos de los casos optan por olvidarse de ellos especialmente cuando estos sufren de discapacidad. Los cuidadores la mayoría de las veces delegan a otras personas o instituciones las responsabilidades de acompañamiento escolar, terapéutica de rehabilitación (López y Loaiza, 2009). En el Ecuador, la Pastoral de Movilidad Humana del Azuay indica que el 20\% de los azuayos ha migrado, de los cuales el 73\% son hombres, el 27\% mujeres (SRJM, 2007).

\subsection{La atención temprana}

Se entiende por Atención Temprana el conjunto de intervenciones, dirigidas por un equipo interdisciplinar a la población infantil de 0-5 años, a la familia y al entorno, que tiene por objetivo dar respuesta lo más pronto posible a las necesidades transitorias o permanentes que presentan los niños con retrasos en su desarrollo o que tienen el riesgo de padecerlos para potenciar la capacidad de desarrollo, de autonomía, de bienestar, posibilitando la inclusión familiar, escolar y social (FEAPS, 1999; Ministerio de Trabajo de Asuntos Sociales de España, 2003). El estudio mexicano indica que las familias no se benefician de la atención temprana porque tienen recelo de comunicar que tienen un hijo o hija con retraso o con discapacidad, existiendo demora en la detección, en el diagnóstico y en el plan de intervención, así en el sector de marginación la media de detección fue entre los 2 años 6 meses y los 7 años, en el grupo de alta marginación entre 4 y 10 años. De los niños y niñas de 0 a 5 años de edad sin diagnóstico, el 33\% de las familias no han buscado ayuda, porque no saben dónde acudir o porque no tienen acceso a los servicios de rehabilitación. Solo el 2,3\% de los niños, niñas y jóvenes con discapacidad recibieron atención integral (Gobierno del Distrito Federal y UNICEF, 2006).

El objetivo general de este estudio transversal fue determinar en los niños y niñas de 0 a 5 años de edad de los Centros de Desarrollo Infantil (CDI) urbanos de Cuenca, la prevalencia de retrasos del desarrollo con o sin discapacidad y su relación con factores asociados. Los objetivos específicos fueron: (i) caracterizar a los niños según edad, sexo, tipo de Centro de Desarrollo Infantil (público regular, público especial, privado regular, privado especial), desarrollo psicomotor (con o sin retraso), beneficio de la atención temprana, estado nutricional (normal, desnutrición global, desnutrición crónica, sobrepeso y obesidad), tipo de familia (nuclear, monoparental, extendida), migración (presente o ausente), tipo de cuidador familiar (madre, padre, abuelos, otros); y (ii) determinar en los 
niños y niñas con y sin retraso del desarrollo psicomotor la presencia de los siguientes factores: desnutrición, microcefalia, familia nuclear, migración de los padres y madre cuidadora.

\section{MATERIALES Y MÉTODOS}

\subsection{Población y entorno}

El universo fue de 8.537 niños y niñas de 0 a 5 años de edad de los CDI urbanos de Cuenca públicos regulares, públicos especiales, privados regulares y privados especiales de enero a julio del 2009. Se consideraron las dificultades de investigaciones anteriores de no encontrar a los niños en sus casas porque estos salen con sus madres o van a los CDI, se hizo un pilotaje previo a la realización de este estudio que consistió en sortear manzanas, casas, visitarlas y buscar a los niños pero se hallaron las mismas dificultades. Estas razones llevaron a identificar como universo de estudio a los niños y niñas que asisten a los CDI. Indudablemente se deja fuera a aquellos que no asisten a los mismos. La decisión de incorporar a los CDI especiales se basó en los siguientes criterios: (i) cumplen con las características de ser centros de cuidado diario; (ii) los niños con discapacidad generalmente son escondidos, si asisten a un centro, no considerarlos, es otra vez invisibilizarlos; (iii) cuando un niño menor de 5 años de edad está en un centro especial es porque buscó previamente uno regular y desde aquí le derivaron; y (iv) son niños que requieren CDI como todos los demás.

Para tener el total del universo, se solicitó a la Dirección de Educación del Azuay, al Instituto de la Niñez y la Familia (INFA) la lista de los CDI; y además, se hizo un recorrido a cada una de las manzanas urbanas de Cuenca ubicando e inventariando a los CDI que no estaban registrados. Mediante una disposición legal, los ministerios solicitaron a los directores que envíen la lista de los niños y niñas de 0 a 5 años con los nombres, fecha de nacimiento y sexo. Se elaboró una base de datos (166 CDI: directores, niños y niñas) que permitió el cálculo muestral. Se consideró la frecuencia de $5 \%$ de retrasos del desarrollo psicomotor, $3 \%$ de error de inferencia, el 95\% de confianza y se calculó la muestra en el StatCalc de Epi Info ${ }^{\mathrm{TM}}$ 3.5.1, consiguiendo 433 niños y niñas de 0 a 5 años de edad, al que se añadió 43 , por el $10 \%$ de pérdidas $(\mathrm{n}=476)$. Se distribuyó por estratos proporcionalmente a como se presentó en el universo, el 28,3\% $(n=135)$ de los centros públicos regulares; $1,2 \%(n=5)$ de los públicos especiales; el 68,51\% $(\mathrm{n}=326)$ de los privados regulares; el $2,17 \%(\mathrm{n}=10)$ de los privados especiales. El sorteo se hizo con el software EpiData (versión 3.0) caracterizando previamente a la población por sexo proporcional a como se presentó en el Universo.

Los criterios de inclusión fueron tener edad entre 0 y 5 años de edad, asistencia regular y que el representante firme el consentimiento informado. El criterio de exclusión fue la actitud persistente de no colaboración del niño o niña. La recolección de los datos se realizó de enero a junio de 2009 , cuando un niño faltó o estuvo enfermo se regresó al otro día para hacer la evaluación y cuando no fue posible se consideró como perdido. Gracias a la colaboración responsable de los maestros, las actuaciones del equipo técnico de investigación (actividades de ambientación, comunicación y buen trato con los niños, padres y maestras que impartió confianza) el número total de niños y niñas que ingresaron a la investigación fue de 462, es decir hubieron 14 casos perdidos.

\subsection{Variables}

La variable dependiente, retraso del desarrollo psicomotor, fue definida como Coeficiente de Desarrollo de 89 o menos, según la metodología de Brunet-Lezine.

Las variables descriptivas fueron las siguientes:

$\checkmark$ Edad, cuantitativa continua, meses y días transcurridos desde la fecha de nacimiento hasta el día de la evaluación.

$\checkmark$ Grupos etarios, cualitativa ordinal: lactantes hasta los 2 años y preescolares de 3 a 5 años.

$\checkmark$ Sexo (diferencia de genitales): masculino y femenino. 
Peso, variable cuantitativa continua: expresada en kilos, medida en balanza bandeja en menores de 12 meses quitándoles toda la ropa y cubriéndolos con una sábana; en balanza de piso de marca Seca para los mayores y en aquellos que no colaboraron o en niños con discapacidad, solo con ropa interior, mediante la "función tara" (reprogramar a cero mientras que una persona recién pesada se para en la balanza y toma al niño) (OMS, 2007 y 2008).

$\checkmark$ Talla, como variable cuantitativa continua: extensión longitudinal del niño en centímetros. Medida en los menores de 2 años en decúbito dorsal en un paidómetro de marca Seca, colocando la cabeza en una línea imaginaria entre el conducto auditivo externo y el borde inferior de la órbita del ojo; los hombros sobre la tabla, la espina dorsal sin arqueo, sujetando las piernas, estirando las rodillas, tomando la medida luego de llevar la pieza de madera hacia los pies planos y con los dedos hacia arriba. Cuando el niño estuvo inquieto y no fue posible mantener ambas piernas en la posición correcta se tomó la medición con una sola pierna. Cuando el niño dobló los dedos y no permitió que la pieza toque las plantas, se hizo un poco de cosquillas haciendo que el niño estire los dedos y deslizando rápidamente la pieza hacia los pies. En los mayores de 2 años en bípedo estación en un tallímetro, con el niño con pies ligeramente separados, cabeza, omóplato, pantorrillas, talón tocando la tabla vertical, la madre sujetando las rodillas y los tobillos, cabeza recta, sujetando la barbilla y registrando la medición. A los niños que no fue posible mantenerlos de pie por su discapacidad se les tomó acostado y se les restó $0,7 \mathrm{~cm}$ (OMS, 2007 y 2008).

$\checkmark$ Índice de masa corporal (IMC), variable cuantitativa continua: de dividir el peso en kilos para la talla en metros al cuadrado (OMS, 2007 y 2008).

$\checkmark$ Perímetro cefálico, operacionalizada como variable cuantitativa continua y como cualitativa ordinal (normocefalia, microcefalia, macrocefalia): circunferencia craneana en centímetros. Medida con cinta flexible de 2 centímetros de ancho que pasa en las partes más sobresalientes: frente y parte prominente del occipucio. Microcefalia: menos de -2 DS. Normocefalia: medición ubicada en las tablas de la OMS y que se ubique entre +2 DS y -2 DS. Macrocefalia: más de +2 DS. También operacionalizada como "microcefalia": sí y no (García y Romero, 2003; OMS, 2007; OMS, 2008).

$\checkmark$ Estado nutricional, variable ordinal: estado de crecimiento determinado por peso/edad, talla/edad, IMC/edad en las tablas de la OMS, son: desnutrición global si peso/edad está bajo -2 DS; desnutrición crónica si talla/edad es -2 DS; normal cuando todos los indicadores están dentro de 2 DS y +2 DS; sobrepeso cuando IMC está entre +2 DS y +3 DS; obesidad cuando IMC está sobre +3 DS (Ministerio de Coordinación de Desarrollo Social, 2007; OMS, 2007 y 2008). También se operacionalizó como "desnutrición": con (cuando alguno de los indicadores de peso, talla, IMC estuvieron por debajo de -2 DS) y sin (cuando todos los indicadores estuvieron dentro de los parámetros normales).

$\checkmark$ Desarrollo psicomotriz, cualitativa: logros en cada etapa medidos por el test de Brunet-Lezine): Retraso con puntuaciones de 89 o menos y sin retraso con 90 o más (Monge, 2002).

$\checkmark$ Coeficiente de desarrollo, variable cuantitativa continua: puntuaciones del Brunet-Lezine.

$\checkmark$ Discapacidad, cualitativa: dependencia para realizar las actividades de la vida diaria de acuerdo a la edad, para niños de 0 a 3 años de edad se empleó la Escala de Valoración Específica de la Situación de Dependencia (EVE) y para niños mayores de 3 años el Baremo de la Situación de Dependencia (BVD). Son clasificados con discapacidad cuando EVE tuvo puntuaciones de 1 a 3 o BVD mayores a 25, y sin discapacidad por puntajes menores al indicado (Gobierno de España Ministerio de Educación, Política y Deporte, 2008).

$\checkmark$ Tipo de familia, cualitativa: institución social cuyos miembros están unidos por lazos de consanguinidad: Monoparental conformada por un solo padre y los niños. Nuclear constituida por los dos padres y los niños. Extendida integrada por más de dos generaciones con niños, padres, abuelos o tíos u otros (Quintero, 2007; Carro y col., 2010). También se operacionalizó como "familia no nuclear": no nuclear (monoparental y expandida) y nuclear (dos padres e niños).

$\checkmark$ Cuidador familiar, cualitativa: persona que principalmente suministra al niño las atenciones de la vida diaria como alimentación, aseo personal, afecto, educación, recreación y participación social: 
madre, padre, abuelos, otros. También se operacionalizó como "cuidadora no materna": no materna (otro cuidador que no sea la madre) y materna (la madre) (Isla, 2000).

$\checkmark$ Migración de los padres, cualitativa: desplazamiento de uno o de los dos padres a una distancia significativa que impida la comunicación y cuidado del niño. Es: presente y ausente (ONU, 2002).

$\checkmark$ Beneficio de la atención, cualitativa: cuando cumple con todos los siguientes: detección, derivación, diagnóstico y plan de intervención. Es: presente y ausente (FEAPS, 1999).

$\checkmark$ Tipo de Centro de Desarrollo Infantil, cualitativa: institución de cuidado diario o de educación inicial a los niños y niñas de 4 a 8 horas diarias. Es: público regular, si el financiamiento procede del estado y atiende a niños sin discapacidad; público especial, cuando el financiamiento es estatal y atiende a niños con discapacidad; privado regular, si el financiamiento proviene de los padres y atiende a niños sin discapacidad; privado especial, si el financiamiento proviene de otras fuentes que no son del estado y atiende a niños con problemas con discapacidad.

Para el análisis de asociación con retraso del desarrollo psicomotor se consideraron las siguientes variables independientes: sexo, desnutrición, microcefalia, migración, familia no nuclear, cuidadora no materna.

\subsection{Instrumentos y procedimientos de recolección de la información}

Se aplicó un formulario con tres secciones: la primera de datos administrativos, la segunda destinada a datos de filiación y la tercera a los datos propios de la investigación. Se anexó a cada formulario las secciones que correspondieron a los test de Brunet-Lezine, EVE, BVD con las instrucciones de evaluación. Previo al inicio de la investigación se realizaron talleres al personal de los CDI. Se preparó y se estandarizó a los equipos en las evaluaciones y llenado de formularios, revisando los materiales e instrumentos a utilizar. Para la recolección de la información y evaluación se conformaron 7 equipos (cada uno con 1 coordinadora investigadora y 2 estimuladoras tempranas). Se estableció una profesional que se encargó de receptar diariamente los formularios de todos los equipos revisando la evaluación de los test, llenado de formularios y corrigiendo a tiempo los errores. La directora de la investigación estableció la comunicación diaria con los CDI, los directores, las maestras, los padres de familia garantizando la logística y la ejecución de la investigación. Se realizó el pilotaje a 40 niños sorteados y que no fueron parte de la muestra. Los equipos hicieron visitas a cada uno de los CDI para solicitar que las maestras ayuden con la firma de los consentimientos informados. Durante 4 meses los 7 equipos salieron cada mañana a los CDI para evaluar a los niños y niñas que constaban en el cronograma. El tiempo aproximado de evaluación de cada niño o niña fue aproximadamente de 2 horas 30 minutos siguiendo los pasos de: primero, llenado con la maestra de los datos de edad, sexo, tipo de familia, migración, tipo de cuidador, beneficio de la atención; segundo, ambientación (juegos, cantos); tercero, medición de antropometría; cuarto, evaluación del desarrollo; quinto, evaluación del nivel de dependencia; sexto, revisión del formulario (que tenga todos los datos). Cada equipo evaluó diariamente a 2 niños por día, se terminó la evaluación en 4 meses. Los test Brunet-Lezine, EVE y BVD fueron calificados en sus puntuaciones el mismo día en la oficina del programa. Como parte del control de calidad se aplicó a 21 casos (5\%), sorteados aleatoriamente de la muestra, una nueva evaluación dentro de los siete días siguientes a la primera. Con los resultados de la evaluación se escribieron informes para cada uno de los niños, niñas y se entregaron a los maestros y padres de familia, derivando a los con retraso del desarrollo a un programa de intervención temprana.

\subsection{Análisis estadístico}

No se encontraron valores perdidos. En el nivel de análisis descriptivo se comprobó (asimetría, curtosis, gráficos QQ plot) que ninguna de las variables cuantitativas continuas (edad en meses, peso en kilos, talla en centímetros, perímetro cefálico en centímetros, IMC, coeficiente de desarrollo) presentó un patrón "normal" y se seleccionó como medidas de tendencia central y de distribución a la mediana, el mínimo, el máximo y los percentiles. Se realizó U de Mann Whitney test para observar la diferencia por sexo de las medianas de estas variables. Se caracterizó a los niños y niñas de la muestra en tablas con frecuencias y porcentajes según: sexo, grupo etario, perímetro cefálico, estado 
nutricional, desarrollo psicomotriz, discapacidad, tipo de familia, cuidador familiar, migración de los padres, tipo de CDI. Análisis por correspondencias para variables cualitativas con más de 3 categorías: tipo de familia con tipo de cuidador. Se obtuvo la prevalencia de los retrasos del desarrollo psicomotor con el intervalo de confianza al 95\% con prueba de una proporción. Para la relación de retraso del desarrollo con las independientes dicotómicas (sexo, grupo etario, desnutrición, microcefalia, migración, cuidadora no materna, familia no nuclear, microcefalia) el chi-cuadrado, razón de prevalencia (RP) con intervalos de confianza al 95\% (IC95\%). Se corrigió con Yates y con el estadístico exacto de Fisher cuando alguna celdilla presentó valores menores a 5 como en el caso de migración y microcefalia. También se realizó regresión lineal multivariada pasos sucesivos con la variable dependiente, coeficiente de desarrollo; y las cuantitativas: peso en kilos, talla en centímetros, perímetro cefálico en centímetros e IMC. En el modelo se dejó fuera los valores atípicos mayores a 2 DS. No se consideró edad en meses porque el cociente de desarrollo medido con Brunet-Lezine no sube con la edad.

\section{RESULTADOS Y DISCUSIÓN}

El derecho al desarrollo integral en los Centros de Desarrollo Infantil para niños y niñas de 0 a 5 años de edad, declaración de la Constitución del Ecuador 2008, no se cumple porque el 71,4\% de los 462 niños y niñas acuden a CDI privados regulares y tan solo el $25,3 \%$ van a fiscales regulares. Los niños y niñas con discapacidad, cuyo cuidado debería ser asumido básicamente por el estado, asisten casi en el mismo porcentaje a los CDI fiscales y privados (Tabla 2). Una limitación de este estudio fue no incorporar a los niños y niñas que se quedan por no tener acceso a un CDI. La UNICEF declara que para el año 2008 en el Ecuador el 46\% de los niños y niñas entre 0 y 5 años de edad no van a CDI (Observatorio Social del Ecuador, 2006; UNICEF, 2008). Las mediana de edad en meses, peso en kilos, talla en centímetros, IMC fue similar tanto en las niñas como en los niños, no así las medianas de coeficiente de desarrollo y perímetro cefálico $(\mathrm{p}<0.05)$ (Tabla 1). Acuden a los CDI de manera

Tabla 1. Características de 462 niños y niñas de los Centros de Desarrollo Infantil Urbanos de Cuenca (2009).

\begin{tabular}{lcccccc}
\hline \multirow{2}{*}{ Variables } & \multicolumn{7}{c}{ Masculino } \\
\cline { 2 - 6 } & Mínimo & Mediana & Máximo & PC 10 & PC 50 & PC 90 \\
\hline Edad (meses) & 8,0 & 49,0 & 59,0 & 24,6 & 49,0 & 58,0 \\
Peso (kg) & 6,2 & 15,0 & 28,0 & 11,3 & 15,0 & 19,0 \\
Perímetro cefálico (cm) & 41,0 & 51,0 & 55,0 & 48,0 & 51,0 & 52,0 \\
Talla (cm) & 63,0 & 100,0 & 114,0 & 84,6 & 100,0 & 107,0 \\
IMC & 10,4 & 15,6 & 23,6 & 13,9 & 15,6 & 18,1 \\
Coeficiente de desarrollo & 11,8 & 102,4 & 149,3 & 88,3 & 102,4 & 118,9 \\
\hline
\end{tabular}

\begin{tabular}{|c|c|c|c|c|c|c|c|}
\hline \multirow{2}{*}{ Variables } & \multicolumn{6}{|c|}{ Femenino } & \multirow{2}{*}{$\mathrm{p}$} \\
\hline & Mínimo & Mediana & Máximo & PC 10 & PC 50 & PC 90 & \\
\hline Edad (meses) & 5,0 & 49,0 & 59,0 & 28,0 & 49,0 & 58,0 & 0,933 \\
\hline Peso (kg) & 5,8 & 15,0 & 22,0 & 11,0 & 15,0 & 19,0 & 0,993 \\
\hline Perímetro cefálico $(\mathrm{cm})$ & 39,0 & 50,0 & 55,0 & 47,0 & 50,0 & 52,0 & 0,001 \\
\hline Talla $(\mathrm{cm})$ & 57,0 & 100,0 & 114,0 & 85,4 & 100,0 & 107,5 & 0,836 \\
\hline IMC & 9,52 & 15,8 & 30,78 & 13,9 & 15,8 & 17,72 & 0,660 \\
\hline Coeficiente de desarrollo & 19,0 & 107,0 & 150,0 & 90,0 & 107,0 & 120,4 & 0,003 \\
\hline
\end{tabular}


Tabla 2. Características de 462 niños y niñas de los CDI Urbanos de Cuenca (2009).

\begin{tabular}{|c|c|c|}
\hline \multirow{2}{*}{ Características } & \multicolumn{2}{|c|}{ Total } \\
\hline & No & $\%$ \\
\hline \multicolumn{3}{|c|}{ Centro de Cuidado Diario } \\
\hline Fiscales regulares & 117 & 25,3 \\
\hline Privados regulares & 330 & 71,4 \\
\hline Fiscales especiales & 6 & 1,3 \\
\hline Privados especiales & 9 & 1,9 \\
\hline Total & 462 & 100,0 \\
\hline \multicolumn{3}{|l|}{ Sexo } \\
\hline Masculino & 245 & 53,0 \\
\hline Femenino & 217 & 47,0 \\
\hline Total & 462 & 100,0 \\
\hline \multicolumn{3}{|l|}{ Grupo etario } \\
\hline Lactantes & 39 & 8,4 \\
\hline Preescolares & 423 & 91,6 \\
\hline Total & 462 & 100,0 \\
\hline \multicolumn{3}{|c|}{ Desarrollo psicomotriz } \\
\hline Con retraso & 51 & 11,0 \\
\hline Sin retraso & 411 & 89,0 \\
\hline Total & 462 & 100,0 \\
\hline \multicolumn{3}{|l|}{ Discapacidad } \\
\hline Con discapacidad & 23 & 5,0 \\
\hline Sin discapacidad & 439 & 95,0 \\
\hline Total & 462 & 100,0 \\
\hline \multicolumn{3}{|c|}{ Migración de los padres } \\
\hline Migración & 24 & 5,2 \\
\hline No migración & 438 & 94,8 \\
\hline Total & 462 & 100,0 \\
\hline \multicolumn{3}{|l|}{ Tipo de familia } \\
\hline Monoparental & 51 & 11,0 \\
\hline Nuclear & 327 & 70,8 \\
\hline Extendida & 84 & 18,2 \\
\hline Total & 462 & 100,0 \\
\hline \multicolumn{3}{|l|}{ Cuidador familiar } \\
\hline Padre & 22 & 4,8 \\
\hline Madre & 385 & 83,3 \\
\hline Abuelos & 41 & 8,9 \\
\hline Otros & 14 & 3,0 \\
\hline Total & 462 & 100,0 \\
\hline \multicolumn{3}{|l|}{ Estado nutricional } \\
\hline Normal & 412 & 89,2 \\
\hline Desnutrición global & 18 & 3,9 \\
\hline Desnutrición crónica & 24 & 5,2 \\
\hline Sobrepeso & 8 & 1,5 \\
\hline Obesidad & 1 & 0,2 \\
\hline Total & 462 & 100,0 \\
\hline \multicolumn{3}{|l|}{ Perímetro cefálico } \\
\hline Normal & 450 & 97,4 \\
\hline Microcefalia & 6 & 1,3 \\
\hline Macrocefalia & 6 & 1,3 \\
\hline Total & 462 & 100,0 \\
\hline
\end{tabular}


similar los niños y niñas y básicamente los preescolares $(91,6 \%)$ ya sea porque culturalmente los lactantes permanecen con sus madres, abuelos o porque varios CDI reciben solo a preescolares.

El porcentaje de niños y niñas con retraso del desarrollo psicomotor es del 11\% (IC95\%, 8 al 14\%), el $6 \%$ sin discapacidad y el $5 \%$ con discapacidad (Tabla 2). Los retrasos se clasifican como leves en el $47 \%$, como moderados en el 35\%, como graves en el 6\%, y como severos en el $12 \%$. El 11\% (IC95\%, 8 al 14\%) de la prevalencia de retraso del desarrollo en nuestro estudio es similar al 12,7\% del estudio de España (Aláez, 1998). Está por debajo otros datos encontrados en la literatura por las siguientes razones:

$\checkmark$ Estudios sobre retraso del desarrollo psicomotor que demuestran frecuencias más altas, han sido indagados en poblaciones específicas como niños y niñas con asfixia, niños de madres con patología en el embarazo, niños con retraso del crecimiento intrauterino, con parálisis cerebral o con malformaciones congénitas (Gottlieb y col., 2009; Laptook y col., 2009; Sathlmann y col., 2009; Gobierno de Chile, 2010).

$\checkmark$ La frecuencia del 24,3\% de la investigación del CEDIUC en Cuenca en el 2002 (Huiracocha $y$ col., 2004) se obtuvo en casos de la consulta de este centro de atención temprana.

$\checkmark$ En el trabajo argentino (PRUNAPE, 2008) la frecuencia del 20\% se establece con la evaluación de niños y niñas que acuden al hospital con la guía PRUNAPE.

La frecuencia de discapacidad en este estudio es más alta que la que reporta el CONADIS $(1,7 \%)$ (2005) y más baja que los datos de niños y niñas con discapacidad entre 2 a 9 años de edad de 18 países reportados por la Encuesta de Indicadores Múltiples por Conglomerados (MICS) de la UNICEF en el 2005 (del 2 al 23\%) (UNICEF, 2010) y los resultados de los censos en Chile, 2004 $(12,9 \%)$ (Gobierno de Chile, 2010). Las grandes diferencias se deben a que los datos son recolectados por preguntas a las familias (CONADIS, 2005; Organización Panamericana de la Salud, 2010) y esta forma de identificar a los niños y niñas con discapacidad no es la más apropiada porque depende del nivel de aceptación, de afrontamiento de las familias. Esta investigación siguió un proceso riguroso de evaluación que permitió una mejor identificación de los niños y niñas con discapacidad.

De los niños y niñas con retraso con o sin discapacidad el $31 \%$ tuvo el beneficio de la atención temprana (detección, derivación, diagnóstico, intervención), datos que concuerdan con el estudio mexicano de la UNICEF del año 2001 al 2003.

En el 5,2\% de la muestra la migración está presente, diferente al 20\% que reporta la Pastoral de Movilidad Humana del Azuay, tal vez se deba a que la investigación se realizó en los CDI urbanos y la migración es más alta en los sectores rurales (SRJM, 2007).

Las familias son sobre todo nucleares $(70,8 \%)$ más que el promedio nacional $(50 \%)$, son monoparentales en el $11 \%$ y extendidas en el $18,2 \%$ (10\% y $35 \%$ respectivamente en el Ecuador). La cuidadora principal sigue siendo la madre $(83,3 \%)$, le siguen los abuelos $(8,9 \%)$, el padre juega un bajo papel en el cuidado $(4,8 \%)$ junto con los otros $(3 \%)$. Resultados similares se han obtenido en un estudio en Brasil de 2007 en donde la madre (88\%) y los abuelos son los cuidadores principales (12\%) (Santa María y Linhares, 2007).

De los 462 niños y niñas evaluados la desnutrición global es del 3,9\%, la desnutrición crónica del 5,2\%. Los datos son más bajos que el 25,8\% de desnutrición crónica del país y del 33,3\% al 40,8\% de Cuenca (Ministerio de Coordinación de Desarrollo Social, 2007). La prevalencia de sobrepeso y obesidad en este estudio fue baja: $1,5 \%$ y $0,2 \%$ respectivamente. Esto se debe a que a los CDI privados asisten niños y niñas con buenas condiciones socioeconómicas y en los públicos se les da 3 comidas al día y tienen un programa de control del crecimiento.

El 1,3\% de los 462 niños y niñas presentó microcefalia, el 1,3\% macrocefalia y el $97,4 \%$ normocefalia.

En el análisis por correspondencias entre tipo de familia y tipo de cuidador se encontró que cuando la familia es nuclear la madre es la cuidadora, cuando la familia es monoparental otros son los cuidadores, cuando es extendida los abuelos son los cuidadores, datos que concuerdan con los reportados por la CEPAL que indica que en América Latina el 41,1\% de familias en el 2005 fueron nucleares siendo la madre la cuidadora porque las estructuras (incremento de la soltería, separaciones, divorcios, migración) sociodemográficas hace que existan nuevas formas familiares como las 
monoparentales y las expandidas en donde los abuelos, los tíos, los vecinos son los encargados del cuidado de los niños y niñas, como una manera de apoyo en la supervivencia (CEPAL, 2007). La relación entre tipo de familia y tipo de cuidador, a través de un análisis multivariante, se presenta en la Fig. 1.

Como se puede observar en la Tabla 3, esta investigación no encontró asociación estadísticamente significativa ni con sexo, ni con migración de los padres, ni con cuidadora no materna. Existe relación significativa con: grupo etario $(\mathrm{p}<0,012)$ porque ser lactante constituye 2,72 veces más riesgo de tener retraso del desarrollo que ser preescolar; con desnutrición $(p<0,001)$ porque tenerla hace que los niños y niñas tengan 3,91 veces más de tener retraso; con microcefalia ( $p$ $<0,001$ ) porque aumenta 17,4 veces más la probabilidad de retraso; con familia no nuclear ( $\mathrm{p}<$ 0,003 ) porque aumenta 2,4 veces más el riesgo de tener retraso del desarrollo psicomotor. Estos datos corroboran lo narrado por otros estudios que marcan la relación desnutrición, microcefalia con retraso del desarrollo psicomotor (Reyes y col., 1990; Ashwal y col., 2009; Gottlieb y col., 2009; Martí y Cabrera, 2010; Wu y col., 2010).

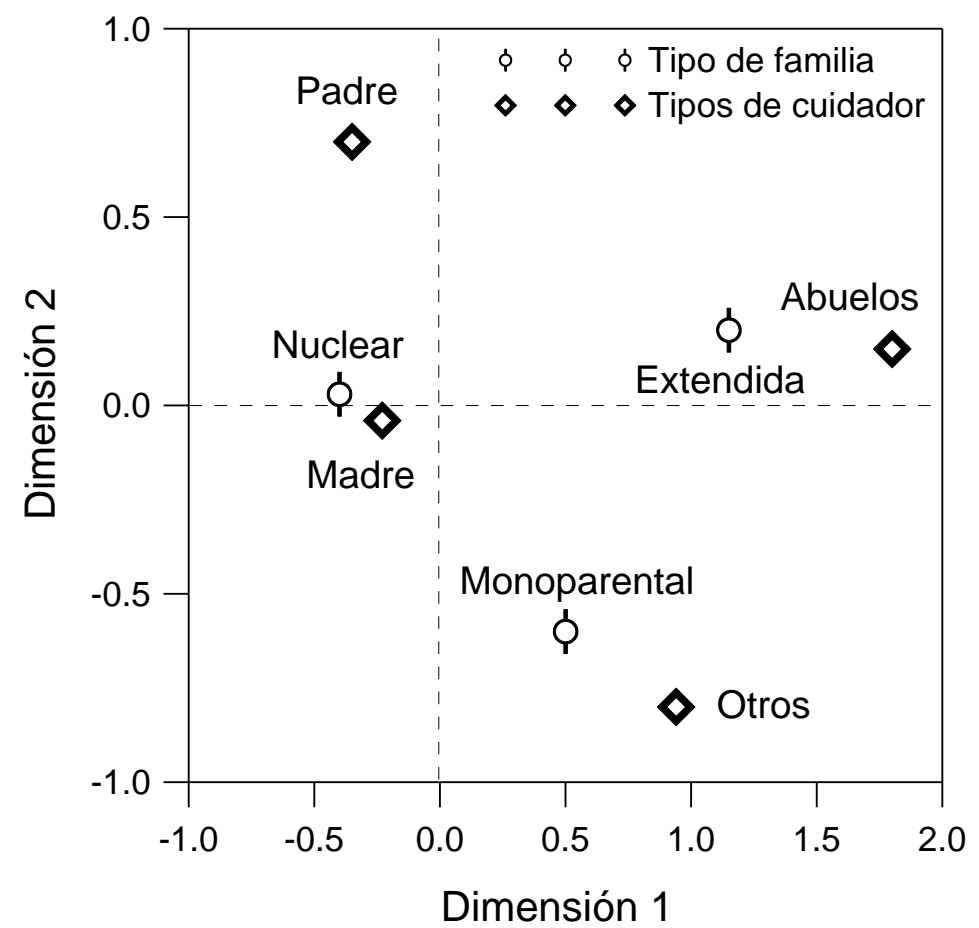

Figura 1. Relación entre tipo de familia y tipo de cuidador.

En regresión lineal multivariada considerando "coeficiente de desarrollo" como dependiente y como independientes a peso en kilos, talla, perímetro cefálico en centímetros y IMC. Se encontró en el modelo: una $p<0,001$, lo que indica que al menos una de las variables introducidas tiene influencia en el coeficiente de desarrollo; con una $\mathrm{R}^{2}$ de 0,108 es decir el 10,8\% de la varianza de la dependiente está explicada por la varianza de las independientes. Se encontró relación de coeficiente de desarrollo solo con perímetro cefálico: por cada 1 centímetro que sube el perímetro cefálico el coeficiente aumenta 0,39 .

Una limitación del estudio es que el número muestral no permitió tener los casos necesarios en migración lo que reduce el poder estadístico. Algo similar ocurre con microcefalia a pesar que en todos los pasos analíticos hay significancia. Se recomienda hacer un estudio de casos y controles para obtener datos más cercanos a la realidad. A pesar de ello, los resultados de la investigación se pueden generalizar a Centros de Desarrollo Infantil Urbanos con similares características. 
MASKANA, Vol. 3, No. 1, 2012

Tabla 3. Factores asociados con retraso del desarrollo psicomotor.

\begin{tabular}{|c|c|c|c|c|c|c|c|c|c|}
\hline \multirow{2}{*}{ Factores asociados } & \multicolumn{2}{|c|}{ Con retraso } & \multicolumn{2}{|c|}{ Sin retraso } & \multicolumn{2}{|c|}{ Total } & \multirow{2}{*}{$p$} & \multirow{2}{*}{$\mathrm{RP}$} & \multirow{2}{*}{ IC95\% } \\
\hline & No & $\%$ & No & $\%$ & No & $\%$ & & & \\
\hline Sexo & & & & & & & 0,379 & 1,30 & $0,70-2,45$ \\
\hline Masculino & 30 & 58,8 & 215 & 52,3 & 245 & 53,0 & & & \\
\hline Femenino & 21 & 41,2 & 196 & 47,7 & 217 & 47,0 & & & \\
\hline Total & 51 & 100,0 & 411 & 100,0 & 462 & 100,0 & & & \\
\hline Grupo etario & & & & & & & 0,012 & 2,72 & $1,12-6,49$ \\
\hline Lactantes & 9 & 17,6 & 30 & 7,3 & 39 & 8,4 & & & \\
\hline Preescolares & 42 & 82,4 & 381 & 92,7 & 423 & 91,6 & & & \\
\hline Total & 51 & 100,0 & 411 & 100,0 & 462 & 100,0 & & & \\
\hline Migración de los padres & & & & & & & 0,507 & 1,16 & $0,26-4,31$ \\
\hline Migración & 3 & 5,9 & 21 & 5,1 & 24 & 5,2 & & & \\
\hline No migración & 48 & 94,1 & 390 & 94,9 & 438 & 94,8 & & & \\
\hline Total & 51 & 100,0 & 411 & 100,0 & 462 & 100,0 & & & \\
\hline Cuidadora no materna & & & & & & & 0,163 & 1,64 & $0,77-3,45$ \\
\hline No materna & 12 & 23,5 & 65 & 15,8 & 77 & 16,7 & & & \\
\hline Materna & 39 & 76,5 & 346 & 84,2 & 385 & 83,3 & & & \\
\hline Total & 51 & 100,0 & 411 & 100,0 & 462 & 100,0 & & & \\
\hline Desnutrición & & & & & & & 0,001 & 3,91 & $1,73-8,72$ \\
\hline $\mathrm{Si}$ & 12 & 23,5 & 30 & 7,3 & 42 & 9,1 & & & \\
\hline No & 39 & 76,5 & 381 & 92,7 & 420 & 90,9 & & & \\
\hline Total & 51 & 100,0 & 411 & 100,0 & 462 & 100,0 & & & \\
\hline Microcefalia & & & & & & & 0,001 & 17,4 & $2,65-141,0$ \\
\hline $\mathrm{Si}$ & 4 & 7,8 & 2 & 0,5 & 6 & 1,3 & & & \\
\hline No & 47 & 92,2 & 409 & 99,5 & 456 & 98,7 & & & \\
\hline Total & 51 & 100,0 & 411 & 100,0 & 462 & 100,0 & & & \\
\hline Familia no nuclear & & & & & & & 0,003 & 2,40 & $1,28-4,52$ \\
\hline No nuclear & 24 & 47,1 & 111 & 27,0 & 135 & 29,2 & & & \\
\hline Nuclear & 27 & 52,9 & 300 & 73,0 & 327 & 70,8 & & & \\
\hline Total & 51 & 100,0 & 411 & 100,0 & 462 & 100,0 & & & \\
\hline
\end{tabular}

\section{CONCLUSIONES}

La investigación permitió determinar que la prevalencia del retraso del desarrollo psicomotor y la discapacidad en niños y niñas de 0 a 5 años de los CDI urbanos de la ciudad de Cuenca es importante, resultados que deben llevar a fortalecer los procesos de atención temprana como cuidado del crecimiento y desarrollo, evaluación, detección, derivación de las banderas rojas del desarrollo, diagnóstico y plan de intervención. La frecuencia alta de discapacidad debe impulsar a que los equipos de salud, de educación y otros profesionales trabajen por la atención de calidad. Los retrasos del desarrollo psicomotor están relacionados con los factores de riesgo de edad en meses del niño, desnutrición, microcefalia hecho que ratifica la necesidad de cuidar la nutrición en los primeros años de vida. Es un factor protector la familia nuclear porque todavía la cuidadora principal sigue siendo la madre. De ahí la importancia de fortalecer este vínculo a través de políticas que den a la mujer mayor oportunidad de participar en el cuidado de sus niños e hijas. 


\section{AGRADECIMIENTOS}

Los autores agradecen a la Dirección de Investigación de la Universidad de Cuenca (DIUC) por el apoyo financiero, al Ing. Vladimiro Tobar, Docente e Investigador de la Facultad de Ingeniería, por la ayuda en el análisis estadístico, y a diferentes administraciones, organismos y personas individuales que para el acceso a la información y datos históricos ha permitido la realización de la investigación, al Concejo Cantonal de la Niñez y Adolescencia, al Ministerio de Salud Púbica, al Ministerio de Educación, al Ministerio de Bienestar Económico y Social, al Instituto de la Niñez y la Familia, a los Centros de Desarrollo Infantil, a las estudiantes de Tecnología Médica, a los padres de familia, a los niños y niñas.

\section{BIBLIOGRAFIA}

Aláez, M., R. Martínez, C. Rodríguez, 1998. Prevalencia de Trastornos Psicológicos en niños y adolescentes, su relación con la edad y el género. Ed. Universidad Complutense, Madrid, España, $50 \mathrm{pp}$.

Asamblea Constituyente, 2008. Constitución 2008. Ministerio de Gobierno, Policía y Cultos, República del Ecuador, 24-25, 33-43.

Ashwal, S., D. Michelson, L. Plawner, W. Dobyns, 2009. Practice Parameter: evaluation of the child with microcephaly (an evidence- based review). Neurology, 73, 887-897.

Avaria, M., 2005. Pediatría del desarrollo y comportamiento. Rev. Ped. Elec. [en línea], 2(1), 88-91.

Bhoomika, R., K. Shobini, L. Rao, A. Chandramouli, 2008. Cognitive development in children with chronic protein energy malnutrition. Behav. Brain. Funct., 4, 31.

Bringiotti, M., 2005. Las familias en "situación de riesgo" en los casos de violencia familiar y maltrato infantil. Texto Contexto Enferm., 14 (número especial), 78-85.

Carro, C., I. Donoso, A. Muela, 2010. Sistemas Familiares: Centros de protección de derechos. MIESINFA, Ecuador, $40 \mathrm{pp}$.

Centro de Atención a la Infancia y a la Familia (CAIF), 1996. Programa de Estimulación Oportuna: Un lugar para crecer y aprender jugando. PLAN CAIF-INAME, Uruguay, $30 \mathrm{pp}$.

CEPAL, 2007. Familias y políticas públicas en América Latina: Una historia de desencuentros. Comisión Económica para América Latina y el Caribe, Chile, 344 pp.

CONADIS (Consejo Nacional de Discapacidades, Instituto Nacional de Estadística y Censos del Ecuador), 2005. La discapacidad en cifras: Análisis de resultados de la encuesta nacional de discapacidades. Programa de Computadora: 1 disco compacto, Ecuador.

Consejo Nacional de la Niñez y Adolescencia, 2007. Agenda Social de la Niñez y Adolescencia 20072010. Juntos por la equidad desde el principio de la vida. CNNA, Ecuador, $20 \mathrm{pp}$.

Culbertson, J., E. Newman, D. Willis, 2003. Desarrollo psicológico en la niñez y la adolescencia. Clínic. Pediátr. Norteam., 4, 721-796.

FEAPS, 1999. Atención Temprana. Orientaciones para la Calidad. FEAPS, Madrid, España, 120 pp.

Fejerman, N., E. Fernández, 1998. Neurología Pediátrica. 2a Edición. Editorial Panamericana, Buenos Aires, Argentina, p. 4-10.

García, J., F. Romero, 2003. Alteraciones del perímetro craneal: microcefalia y macrocefalia. Pediatr. Integral, VII (8), 587-600.

Gobierno de Chile, 2004. Prevalencia de la discapacidad en Chile. Descargado de: http://www.ine.cl/canales/chile_estadistico/encuestas_discapacidad/pdf/presentacionresultadoses tudionacionaldeladiscapacidad.pdf el 24 de marzo de 2011. 
Gobierno del Distrito Federal (DIF-DF) y UNICEF, 2006. El reto de la Inclusión y Atención Integral de niños, niñas y jóvenes con discapacidad en el Distrito Federal. México. Descargado de: http://www.unicef.org/mexico/spanish/mx_resources_reto_inclusion_estudio_discapacidad.pdf el 30 de marzo de 2010.

Gobierno de España, Ministerio de Educación, Política y Deporte, 2008. Baremo de Valoración de los Grados y Niveles de Dependencia (BVD). SID, Servicio de información para Discapacidad. Descargado de: http://sid.usal.es/idocs/F8/FDO19028/manual_uso_bvd.pdf el 17 de febrero de 2008.

Gobierno de España, Ministerio de Educación, Política y Deporte, 2008. Manual para el uso de la Escala Específica de Dependencia para personas menores de 3 años de edad (EVE). SID, Servicio de información para Discapacidad. Descargado de: http://sid.usal.es/idocs/F8/ FDO19027/manual_de_uso_eve.pdf el 17 de febrero de 2008.

Gottlieb, C., M. Maenner, C. Cappa, M. Durkin, 2009. Child disability screening, nutrition, and early learning in 18 countries with low and middle incomes: data from the third round of UNICEF's Multiple Indicator Cluster Survey (2005-06). Lancet, 374 (9704),1831-9.

Huiracocha, L., M. Huiracocha, F. Palacios, 2004. Frecuencia del retraso del Desarrollo Psicomotor en 284 ninos y niñas menores de 6 años de edad del CEDIUC de la Universidad de Cuenca (2002). Revista de la Facultad de Ciencias Médicas de la Universidad de Cuenca, 26, 53-58.

Isla, P., 2000. El cuidador familiar. Una revisión sobre la necesidad del cuidado doméstico y sus repercusiones en la familia. Cultura de los Cuidados, IV(7,8), 187-194.

Lahiri, S., S. Mukhopadhyay, K. Das, S. Ray, D. Biswas, 1994. Study of impact of epidemiological factors on intelligence of rural childrens of 3 to 6 year age group belonging to low socioeconomic status. Indian J. Public Health, 38(4),133-42.

Larrea, R., 2008. Mapa de las Instituciones Públicas y Privadas en Protección Especial en niños, niñas y adolescentes. Informe final de consultoría. INNFA, Quito, Ecuador.

Laptook, A.R., S. Shankaran, N. Ambalavanan, C. Wa, S.A. McDonald, R.D. Higgins, A. Das, 2009. Outcome of term infants using apgar scores at 10 minutes following hypoxic-ischemic encephalopathy. Pediatr., 124(6), 1619-26.

López, L., M. Loaiza, 2009. Padres o madres migrantes internacionales y su familia: Oportunidades y nuevos desafíos. Rev. Latinoam. Cienc. Soc. Niñez, 7(2), 837-860.

Mansur, S., S. Neto, 2006. Desenvolvimiento neuropsicomotor de lactantes desnutridos. Rev. Bras. Fisioter., 10(2), 185-196.

Martí, M., J. Cabrera, 2010. Macro y microcefalia. Trastornos del crecimiento craneal. Descargado de: http://www.aeped.es/sites/default/files/documentos/25-macromicrocefalia.pdf el 26 de noviembre de 2011.

Ministerio de Coordinación de Desarrollo Social, 2007. Mapa de la Desnutrición Crónica en el Ecuador. FESAECUADOR S.A., Quito, Ecuador, 170 pp.

Ministerio de Trabajo de Asuntos Sociales de España, 2003. Libro Blanco de la Atención Temprana. $4^{a}$ Edición. Real patronato de discapacidad, Madrid, España, p. 13-15.

Monge, M., 2002. Instrumentos de evaluación del desarrollo motor. Educación, 26(1), 155-168.

Narbona, J., E. Schlumberger, 2008. Retraso psicomotor. Asoc. Españ. Pediatr., 21, 152-157.

Nelson, A., C. Vargas, 2001. Cambios en la familia: repercusiones en la práctica pediátrica. Rev. Chil. Pediatr., 72(2), 77-80.

Observatorio de los Derechos de la Niñez y Adolescencia, 2010. Los niños y niñas del Ecuador a inicios del siglo XXI. Secretaria Técnica del Observatorio, Ecuador, 66 pp.

Observatorio Social del Ecuador, 2006. Estado de los Derechos de la Niñez y la Adolescencia en el Ecuador. UNICEF, Quito, Ecuador, 265 pp.

Oiberman, A., L. Orellana, M. Mansilla, 2006. Evaluación de la inteligencia en bebés argentinos: Escala Argentina de Inteligencia Sensoriomotriz. Arch. Argent. Pediatr., 104(4), 316-324. 
OMS, 2001. Clasificación Internacional del Funcionamiento de la Discapacidad y de la Salud. Organización Mundial de la Salud. Editorial Grafo, Madrid, España. Descargado de:

http://ccp.ucr.ac.cr/bvp/pdf/desarrollohumano/oms-clasificacion-01.pdf el 25 de octubre de 2008.

OMS, 2007. Midiendo el crecimiento de un niño. Curso de capacitación sobre la evaluación del crecimiento del niño. Organización Mundial de la Salud. Descargado de: http://www.who.int/childgrowth/training/b_midiendo.pdf el 15 de febrero de 2009.

OMS, 2008. Interpretando los indicadores de crecimiento. Curso de capacitación sobre la evaluación del crecimiento del niño. Organización Mundial de la Salud. Descargado de: http://www.who.int/childgrowth/training/c_interpretando.pdf el 15 de febrero de 2009.

ONU, 2002. Reporte Migración Internacional (documento ESA/P/WP.178), de la División de Población del Departamento de Asuntos Económicos y Sociales de la ONU. Las migraciones contexto actual. Descargado de: http://www.unpopulation.org el 12 de febrero de 2009.

Organización Panamericana de la Salud, 2010. Salud de las Personas con discapacidad - Chile. Descargado de: http://new.paho.org/chi/index.php?option=com_content\&task $=$ view\&id $=82 \&$ Itemid $=259$ el 24 de marzo de 2010 .

Peñafiel, F., A. Hernández, A. Chacón, 2003. Atención temprana. Enseñanza, 21, 245-274.

Perales, C., E. Heresi, F. Pizarro, M. Colombo, 1996. Las nociones cognitivas en niños con CI normal y grave desnutrición. Arch. Latinoam. Nutr., 46(4), 282-286.

Polit, D., 2008. Marco conceptual de la protección especial en niños, niñas y adolescentes. Informe final de consultoría INNFA, Quito, Ecuador.

PRUNAPE, 2008. Pesquisa de trastornos del desarrollo Psicomotor en el Primer Nivel de Atención. Arch. Argent. Pediatr., 106(2), 119-125.

Quintero, A., 2007. Diccionario Especializado en Familia y Género. 1ª Edición. Ed. Lumen, Buenos Aires, Argentina, $65 \mathrm{pp}$.

Reyes, M.R., C.M. Valdecanas, O.L. Reyes, T.M. Reyes, 1990. The effects of malnutrition on the motor, perceptual and cognitive functions of Filipino children. Int. Disabil. Stud., 12(4), 131-136.

Robaina, G., V. Rodríguez, 2000. Comportamiento del desarrollo sicomotor en el menor de 1 año, en relación con el manejo y funcionamiento familiar. Rev. Cubana Med. Gen. Integr., 16(6), 540544.

Santa María, M., M. Linhares, 2007. Factores de Riesgo para problemas de desarrollo infantil. Rev. Latino-am Enferm., 15(número especial), 6 pp.

Secretaría Técnica Del Frente Social, 2004. Plan Nacional Decenal de Protección Integral a la Niñez y Adolescencia. Consejo Nacional de la Niñez y Adolescencia, 109 pp. Descargado de: http://plan.senplades.gob.ec/ el 23 de agosto de 2010.

SRJM, 2007. Percepciones de niños, niñas y adolescentes sobre el hecho migratorio en el Sistema Educativo Formal. Servicio Jesuita de Refugiados y Migrantes del Ecuador. Descargado de: http://www.sjrmecuador.org.ec/portal/files/Estudio\%20Percepciones\%20de\%20NNA\%20y\%20m igraci\%C3\%B3n.pdf el 31 de diciembre de 2011.

Sathlmann, N., M. Rapp, E. Herting, U. Thyen, 2009. Outcome of extremely premature infants at early school age: health-related quality and neurosensory, cognitive, and behavioral outcomes in population-based simple in northern Germany. Neuropediatrics, 40(3), 112-9.

UNICEF, 2008. Supervivencia Infantil en el Ecuador: Avances y desafíos. 2008. Programa de Computadora: 1 disco compacto, Quito, Ecuador.

UNICEF, 2010. Niños con discapacidades. Descargado de: http://www.unicef.org/spanish/progressforchildren/2007n6/index_41853.htm el 24 de marzo 2010.

Upadhyay, S., D. Agarwal, K. Agarwal, 1989. Influence of malnutrition on intellectual development. Indian J. Med. Res., 90, 430-41.

Wu, L., J. Katz, L.C. Mullany, E. Haytmanek, S.K. Khatry, G. Darmstadt, K.P. West Jr., S.C. LeClerq, J.M. Tielsch, 2010. Association between nutritional status and positive childhood 
MASKANA, Vol. 3, No. 1, 2012

disability screening using the ten questions plus tool in Sarlahi, Nepal. J. Health Popul. Nutr., 28(6), 585-594. 